\title{
Object clitics and clitic climbing in Italian HPSG grammar
}

\author{
Paola Monachesi * \\ Tilburg University - CLS/ITK \\ Postbus 90153, 5000 LE Tilburg, Nederland \\ e-mail: paola@kub.nl
}

\section{Introduction}

Italian object clitics can be involved in nonlocal dependencies in the sense that they must/may appear on a verbal head of which they are not an argument. Two cases where this situation arises will be discussed: the first is due to the presence of an auxiliary verb and the second is triggered by the presence of a certain class of verbs that allows clitic climbing.

An analysis will be proposed within the framework of Head-driven Phrase Structure Grammar [Pollard and Sag, 1987; Pollard and Sag, 1993]; it can be shown that an analysis in terms of nonlocal features and the Nonlocal Feature Principle, which is the mechanism provided by HPSG to deal with nonlocal dependencies, does not provide a satisfactory account of the phenomenon; it is too powerful. An alternative approach will be proposed; it is based on the idea that the arguments of a verb which is governed by an auxiliary or clitic climbing trigger verb can be raised to become arguments of the governor by a mechanism that achieves an effect similar to functional composition as developed within the tradition of Categorial Grammar. This approach is able to capture the right generalizations, to account for the relevant data and can be easily extended to account for long NP-movement which is another property of clitic climbing trigger verbs.

\section{Some linguistic properties of Italian clitics}

There are arguments similar to the ones proposed by [Miller, 1992a] for French showing that Italian clitics exhibit many properties that make them very similar to inflectional affixes; the arguments are mainly based on [Zwicky and Pullum, 1983]. The coordination criteria proposed by [Miller, 1992b] can also be used to support this position.

Italian clitics exhibit a high degree of selection with respect to their host; they can only attach to verbs.

They present arbitrary gaps in their combination, since not all the combinations are allowed, behaving therefore like affixes; in particular, it is not possible to have a first or second person accusative clitic together with a dative one.

Italian clitics also exhibit morphophonological idiosyncrasies: vowel deletion occurs when clitics like lo and la occur in front of a vowel initial stem. This applies also to mi, ti, ci, vi, li and si; but mainly in

\footnotetext{
"Supported by a grant from the Center for Language Studies (CLS).
}

spoken language. Certain changes also occur when more than one clitic is present, namely the final -i of a clitic is changed into -e if it is followed by another clitic which begins with 1- or n-. Sequencing of identical clitics is not permitted in Italian, therefore certain changes occur as in the combination of two si or of two vi where one becomes ci. Furthermore, if the third person dative feminine clitic le precedes a clitic beginning with 1- or n-, the masculine dative form gli is used instead of the feminine one.

Other evidence for the affixal status of clitics comes from coordination. Italian clitics cannot have wide scope over coordination of verbs; the following is not possible:

$$
\begin{aligned}
& \text { * Maria lo comprera'e leggera' } \\
& \text { Maria cl.(acc) will buy and will read } \\
& \text { 'Maria will buy and read it' }
\end{aligned}
$$

The clitic should be repeated in front of each verb behaving like an affix according to the coordination criterium developed by [Miller, 1992b].

Italian clitics are rigidly ordered in a flat clitic cluster, adhering to the following ordering:

(2) Clitics order in Italian

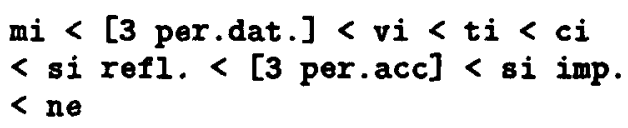

Rigid ordering of elements has often been related to the status of morphological affix.

The data presented above show evidence in favour of an analysis of Italian pronominal clitics as inflectional affixes; ${ }^{1}$ this will be the assumption underlying both of the analyses which will be presented in the following sections.

As already mentioned above, Italian clitics cluster around the verb; they precede it if the verb is finite and follow it if the verb is non-finite or an imperative.

If there is an auxiliary, the clitic doesn't attach to the verb that subcategorizes for it, but cliticizes to the auxiliary as in:

(3) Maria l' ha mangiato Maria cl.(acc) has eaten

'Maria has eaten it'

\footnotetext{
${ }^{1}$ Italian clitics can appear both in proclitic and in enclitic position; it could be argued that this alternation is not typical of affixes. Data from Afar, Swazi and Arabic show that in these languages there are dual position affixes.
} 
If in the main clause there is a restructuring verb [Rizzi, 1982], namely a verb which belongs to one of the following classes: modals, temporal aspectuals, pure motion verbs, the clitic can attach to the main verb, but it can also attach to the verb in the embedded sentence:

$$
\begin{aligned}
& \text { a. Maria lo vuole comprare } \\
& \text { Maria cl.(acc) wants to buy } \\
& \text { 'Maria wants to buy it' } \\
& \text { b. Maria vuole comprarlo } \\
& \text { Maria wants to buy cl.(acc) } \\
& \text { 'Maria wants to buy it' }
\end{aligned}
$$

If there is more than one verb that belongs to one of the classes mentioned above, the clitic can attach to the lower verb or climb to the middle position or all the way up.

Since clitic climbing is triggered by the restructuring verbs, the following will be ungrammatical because the main verb does not belong to this class:

$$
\begin{aligned}
& \text { * Maria lo decide di leggere } \\
& \text { Maria cl.(acc) decides to read } \\
& \text { 'Maria decides to read it' }
\end{aligned}
$$

As for their distribution, Italian clitics are in complementary distribution with full phrases as complements of a lexical head; so a sentence like the following will not be grammatical: ${ }^{2}$

$$
\begin{aligned}
& \text { * Maria lo da' il libro a Giovanni } \\
& \text { Maria cl.(acc) gives the book to Giovanni } \\
& \text { 'Maria it gives the book to Giovanni' }
\end{aligned}
$$

\section{A treatment in terms of nonlocal features}

As was shown by the examples in the previous section, in certain cases a clitic corresponding to the complement of a head is not present on that head, but on a higher node: clitics can thus be involved in nonlocal dependencies. HPSG has a syntactic mechanism to account for Unbounded Dependency Constructions, namely the Nonlocal Feature Principle and the use of NONLOCAL features, which are analogous to the Foot Feature Principle and to the FOOT features used in GPSG [Gazdar et al., 1985]. Therefore it seems a reasonable choice to use the same mechanism to handle cases of nonlocal dependencies involving clitics. ${ }^{3}$

As discussed above, Italian pronominal clitics are in complementary distribution with full phrases as

\footnotetext{
${ }^{2}$ If the full phrase is left (or right) dislocated, its cooccurrence with a clitic pronoun is possible. See [Sanfilippo, 1990] for an analysis of this type of constructions within the Unification Categorial Grammar framework.

${ }^{3}$ See [Monachesi, 1992] for a more detailed description of the analysis and [Miller, 1992a] for a similar analysis of related facts in French within a GPSG/HPSG framework.
}

complements of a lexical head; they must therefore fulfill the subcategorization requirements of the head of which they are a semantic argument. Furthermore, the information that the clitic will appear at some point in the tree must be encoded if a verb that triggers clitic climbing is present.

A lexical rule can be used for this purpose; ${ }^{4}$ it will operate on the subcategorization list, removing the slot related to the relevant full complement and adding a nonlocal feature OC (object clitic) which encodes case and agreement information of the clitic.

Since this is a nonlocal feature, its value can percolate up the tree according to the Nonlocal Feature Principle [Pollard and Sag, 1993]:

(7) Nonlocal Feature Principle For each nonlocal feature, the INHERITED value on the mother is the union of the INHERITED values on the daughters minus the TO-BIND value on the head daughter.

A TO-BIND|OC feature is assigned to the cliticized verb form and will have as its value the agreement and case information relative to the clitic. If there is identity of values between the TO-BIND|OC feature and the INHERJOC feature the percolation of the latter will be stopped and the dependency will be bound off. Therefore, for a sentence like (4a), the following representation will be produced:

(8) Example of derivation

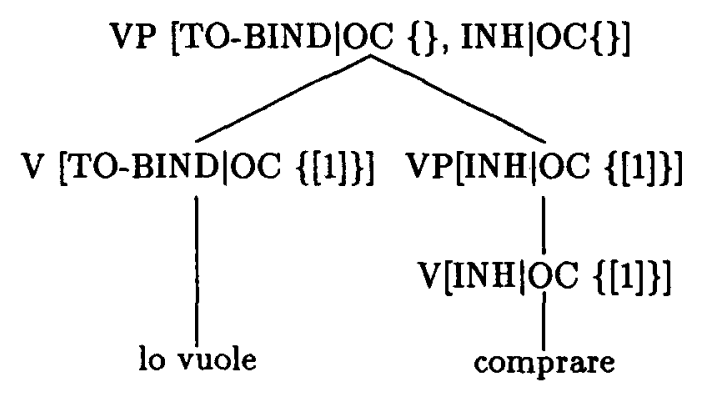

This treatment can account for the following cases:

- cases with an auxiliary: the clitic must climb in order to attach to it;

- cases with a verb that allows clitic climbing: the clitic may climb to attach to it.

However, the mechanism as it has been sketched so far will overgenerate allowing clitic climbing also with verbs that do not trigger it; a sentence like (5) will be accepted. It is necessary to add specific locality constraints on the path of the $\mathrm{OC}$ feature in order

\footnotetext{
${ }^{4}$ In the framework developed in Chapter 9 of [Pollard and Sag, 1993], lexical rules are also used in the treatment of Unbounded Dependency Constructions to produce an analysis that doesn't make use of empty categories.
} 
to make the right predictions, namely the percolation of the feature must be stopped if there is a verb that doesn't trigger clitic climbing and must be allowed if there is a verb that triggers clitic climbing. It seems therefore that this approach doesn't naturally capture the fact that clitic climbing is triggered only by a specific class of verbs since constraints need to be imposed to obtain this result. This fact can be easily captured by an approach in terms of functional composition which will be described in the next section.

\section{A functional composition approach to clitic climbing}

The idea that underlies the approach was originally presented by [Hinrichs and Nakazawa, 1990] for the treatment of the German verb cluster. They argue that the arguments of a verb which is governed by an auxiliary can be raised to become arguments of the auxiliary. They achieve this by making crucial use of the notion of structure sharing which is characteristic of unification-based formalisms such as HPSG. The effect is similar to functional composition as developed within Categorial Grammar.

This approach can be extended to clitics in order to account for the clitic climbing cases producing an analysis which captures intuitions similar to the ones underlying a restructuring analysis [Rizzi, 1982] and a clause reduction one [Aissen and Perlmutter, 1983]. In this case, the arguments of a verb which is governed by an auxiliary or clitic climbing trigger can be raised to become arguments of the governor. It will be shown that the approach can handle the relevant data concerning clitic climbing adequately and that it can account naturally for the fact that only certain verbs can trigger clitic climbing. Furthermore, it can easily be extended to account for another property of restructuring verbs, namely the possibility of allowing for long NP-movement as in [Rizzi, 1982].

\subsection{The analysis}

The analysis is based on the assumption, previously motivated, that Italian clitics behave in a way similar to inflectional affixes. This implies that the verb forms a unit with the clitic and such combination should be accounted for in the morphological module. An account in terms of template morphology as in [Simpson and Withgott, 1986] could handle the rigid ordering of the clitics and the restrictions in combination.

As discussed above, clitics and full complements are in complementary distribution in Italian: the clitic should fill the relevant slot in the subcategorization requirements so that no full complement can occur. On the assumption that clitics behave like inflectional affixes and not as syntactically independent elements, it is necessary to have a way to remove the subcategorization slot related to the full complement if a clitic is present. The Valence princi- ple, which is the principle of the grammar responsible for checking off subcategorization requirements that have been satisfied, cannot be used in this case since the clitic doesn't have the status of a syntactic element. ${ }^{5}$ A lexical rule (LRCL1) can be used instead; namely given a verb that subcategorizes for a certain argument, the argument is removed from the subcategorization list but the equivalent clitic must be present, attached to the verb as proposed by [Miller and Sag, 1993] for French. The lexical rule triggers the presence of the clitic as verb inflection and acts as an interface to morphology. It should look roughly as follows:

(9) Input of lexical rule (LRCL1)

$$
\left[\begin{array}{l}
\text { HEAD V } \\
\operatorname{COMPS}\langle\ldots, X, \ldots\rangle \\
\operatorname{CLTS} W
\end{array}\right]
$$

(10) Output of lexical rule (LRCL1)

$$
\left[\begin{array}{l}
\text { HEAD V } \\
\text { COMPS }\langle\ldots\rangle \\
\text { CLTS W U }\{\mathrm{X}\}
\end{array}\right]
$$

Therefore, when the clitic attaches to the verb that subcategorizes for it, it cliticizes on the host in the morphological component and the relevant slot is removed from the subcategorization list by means of the lexical rule.

When clitics are involved in nonlocal dependencies, namely when the clitic attaches to a head which doesn't subcategorize for it, the argument raising comes into play in addition to LRCL1.

This analysis will look at two cases where this situation arises: the case where an auxiliary verb is present and the case where a restructuring verb is present.

In this treatment, auxiliaries and verbs that trigger clitic climbing subcategorize for a non-finite VP complement and for the complements of the VP; the construction of a partial VP must therefore be allowed. ${ }^{6}$

\footnotetext{
${ }^{5}$ The analysis is carried out within the framework of Chapter 9 of [Pollard and Sag, 1993] which incorporates innovations due to Borsley [Borsley, 1987; Borsley, 1989]. The analysis makes use of valence features which encode the subcategorization requirements of the sign.

${ }^{6}$ This implies the parameterization for Italian of Schema 2 in order to allow partially saturated phrases. (Compare [Pollard, 1990]). Schema 2 describes phrases consisting of a lexical head daughter and any number of complement daughters. One problem which is related to having a VP as complement is that of spurious ambiguity if a non clitic complement is present. The possibility of having a $\mathrm{V}$ as complement instead of a VP is under investigation; see also [Rizzi, 1982] which postulates the presence of a $\mathrm{V}$ if restructuring has applied. Under this
} 
Auxiliaries should have a lexical entry like the following (only relevant features are mentioned): ${ }^{\text {? }}$

(11) Lexical entry for auxiliary verb

$$
\left[\begin{array}{l}
\text { HEAD V [+ AUX] } \\
\operatorname{SUBJ}\langle 1] N P[\text { nom }]\rangle \\
\operatorname{COMPS}\langle 2] \vee P\left[\begin{array}{l}
{[-\mathrm{CL}]} \\
\mathrm{SUBJ}\langle[1] N P\rangle \\
\operatorname{COMPS} L
\end{array}\right]
\end{array}\right]
$$

The [-CL] restriction prevents an auxiliary from combining with a VP whose head has already combined with a clitic, since all cliticized verb forms are specified as $[+C L]$ in the lexicon. In the case of auxiliaries, clitic climbing is obligatory and a sentence like the following must be ruled out:

$$
\begin{aligned}
& \text { * Maria ha lettolo } \\
& \text { Maria has read cl.(acc) } \\
& \text { 'Maria has read it' }
\end{aligned}
$$

The [-CL] lexical specification on the VP complement selected by the auxiliary achieves this purpose; lettolo will be marked as [+CL] and will consequently not meet the specifications of the lexical entry for auxiliaries which forces the verbal complement to be [-CL]. Sentence (12) will be correctly ruled out. The combination of a clitic with a past participle cannot be ruled out in general in Italian, since the following sentence is grammatical:

(13)
Vistolo, fu facile decidere Seen $_{\text {past.part }}$ cl., was easy to decide 'Having seen him, it was easy to decide'

It is only when the past participle combines with the auxiliary that the clitic must attach to the latter.

As for semantic role assignment, it proceeds in a very straightforward way. In HPSG roles are assigned within the lexical entry: a role is assigned by means of structure sharing between the index of an element in the SUBJ/COMPS list and the value of some attribute of the verb's CONTENT value. Role assignment, as well as case assignment, occurs within the lexicon. If a clitic is present, it will be assigned semantic role and case through the link created via structure sharing.

An example will be given to illustrate the mechanism and the interaction of the two lexical rules in

hypothesis it won't be necessary to parameterize Schema 2 and the spurious ambiguity will be avoided, at least with auxiliaries, if all complements of the past participle are forced to raise. See [Abeille and Godard, 1993] for a similar solution for French. Furthermore, the use of the [-CL] feature (see below) imposed on the complement of a restructuring verb is also superfluous under the assumption that all the complements of $\mathrm{V}$ should raise.

${ }^{7}$ Here L stands for the list of complements subcategorized by the lower verb. the analysis of a sentence like (3). In this case the verb ha has the following complement list:

(14) Example of the complement list of ha

COMPS < VP [COMPS <MP [acc]>], MP [acc] >

The auxiliary verb takes a VP and the NP argument of the VP which was not realized within it. This will undergo LRCL1 to produce the following for the cliticized verb form l'ha, where the slot related to the NP is removed from the subcategorization requirement of the auxiliary and the presence of the clitic is imposed:

\section{(15) Example of the complement list of l' ha}

\section{COMPS < VP [COMPS 〈NP [acc]>] >}

Clitic climbing with restructuring verbs can be handled by the same mechanism, but in this case the climbing of the clitic is optional. In such a situation a lexical rule can be used; given the following input (only relevant features are mentioned):

(16) Input of lexical rule (LRCL2)

$$
\left[\begin{array}{l}
\text { HEAD V } \\
\text { VCLASS modal; aspectual;motion } \\
\text { SUBJ }\langle N P[1\rangle \\
\operatorname{COMPS}\langle 2] V P\left[\begin{array}{l}
\operatorname{SUBJ}\langle N P \\
\operatorname{COMPS} L
\end{array}\right]
\end{array}\right]
$$

This will be the output produced (only relevant features are mentioned):

(17) Output of lexical rule (LRCL2)

$$
\left[\begin{array}{l}
\text { HEAD V } \\
\text { VCLASS modal;aspectual;motion } \\
\operatorname{SUBJ}\langle N P[1] \\
\operatorname{COMPS}\langle 2] V P\left[\begin{array}{l}
{[-\mathrm{CL}]} \\
\operatorname{SUBJ}\left\langle N P_{1}\right\rangle \\
\operatorname{COMPS} L
\end{array}\right]
\end{array}\right]
$$

The left hand side (input) of the lexical rule accounts for cases where the clitic doesn't climb as in sentence (4b); in this case there is no argument raising and LRCL1 applies, checking off the slot related to the full complement and triggering the cliticized verb form. If clitic climbing occurs as in (4a), there is argument raising and the right hand side (output) of the lexical rule will account for it; in this specific case the restructuring verb will subcategorize for the verbal complement and for the argument of the verbal complement. Again LRCL1 will trigger the cliticized verb form.

Clitic climbing trigger verbs also subcategorize for a VP [-CL]; this avoids sentences where there are 
two clitics with one attaching to the lower verb and the other to the higher one. In Italian, if there are two clitics that originate as complements of the same verb, they must cliticize together:

a. Piero voleva darmelo Piero wanted to give cl.(dat) cl.(acc) 'Piero wanted to give it to me'

b. Piero me lo voleva dare Piero cl.(dat) cl.(acc) wanted to give 'Piero wanted to give it to me'

The clitics cannot be split:

$$
\begin{aligned}
& \text { * Piero lo voleva darmi } \\
& \text { Piero cl.(acc) wanted to give cl.(dat) } \\
& \text { 'Piero wanted to give it to me' }
\end{aligned}
$$

The restriction on the VP doesn't allow argument raising if the VP already has a clitic as its daughter. In the specific case of sentence (19), darmi will be marked as [+CL] and will not match the requirements imposed by the right hand side of LRCL2 therefore no argument raising will take place and the sentence will be correctly ruled out. As for (18a) and (18b) they will be handled by the left hand side and by the right hand side of LRCL2 respectively. Lexical rule LRCL1 will be responsible in both cases for the cliticized verb form.

If there is more than one restructuring verb, one can get sequences like the following, where the clitic is attached to the higher verb:

$$
\begin{aligned}
& \text { Maria lo vuole poter comprare } \\
& \text { Maria cl.(acc) wants can buy } \\
& \text { 'Maria wants to be able to buy it' }
\end{aligned}
$$

The application of lexical rule LRCL2 is therefore triggered twice so that the higher verb will subcategorize also for the complement of the lower one. Lexical rule LRCL1 will be responsible for the cliticized verb form.

An advantage of this approach is that the possibility of subcategorizing for the complements of the lower VP is restricted to auxiliary and clitic climbing trigger verbs. Therefore a sentence like (5) will be ruled out since the main verb is not lexically marked as a clitic climbing trigger and will not undergo lexical rule LRCL2 which allows argument raising. In this way the fact that clitic climbing occurs only with certain verbs can be naturally captured by the mechanism; only auxiliary verbs and restructuring verbs will trigger argument raising and no special locality constraints are necessary.

\subsection{Extensions}

Restructuring verbs as in [Rizzi, 1982] not only have the property of allowing clitic climbing, they also allow long NP-movement as in:
(21) Queste case si vogliono vendere a caro These houses SI want to sell at high prezzo price

'These houses are wanted to be sold at high price'

In this kind of construction the object of the lower verb, queste case, is allowed to raise and become the subject of the main verb; this option being available only with restructuring verbs. It can be shown that a functional composition approach can easily account for this kind of construction. ${ }^{8}$ Lexical rule LRCL2 allows raising of the NP object of the lower VP which becomes a complement of the restructuring verb. The clitic si acts as a passivizing element in this kind of construction and will trigger the HPSG mechanism that handles passive constructions; namely a lexical rule that acts on the subcategorization requirements of the relevant verb, making the object become the subject as in [Pollard and Sag, 1987]. The NP object will therefore become the subject of the restructuring verb and agreement will be handled by the usual mechanism responsible for subject-verb agreement.

\section{Conclusion}

An analysis for the treatment of clitic climbing in Italian has been proposed. It relies on the idea that auxiliaries and clitic climbing trigger verbs subcategorize for a VP and for the raised complements of the VP. This analysis allows a unified treatment for the simple cases, namely when a clitic attaches to a host that subcategorizes for it and for the cases where clitics are involved in nonlocal dependencies. It has been shown that the analysis rules out illformed sentences. It is not necessary to state specific constraints that would be required in a treatment based on nonlocal features and the Nonlocal Feature Principle, which is another possible way to handle nonlocal dependencies in HPSG. Furthermore, the approach can easily account for another property of restructuring verbs namely long NP-movement.

\section{Acknowledgments}

I am grateful to Anne Abeille, Hap Kolb, Philip Miller, Ivan Sag, Wietske Sijtsma and to a number of collegues from ITK and GM for comments and discussion.

\section{References}

[Abeille and Godard, 1993] A. Abeille and D. Godard. The Syntactic Structure of French Auxiliaries. In preparation, 1993.

\footnotetext{
${ }^{8}$ See [Monachesi, 1993] for further details.
} 
[Aissen and Perlmutter, 1983] J. Aissen and D. Perlmutter. Clause Reduction in Spanish. In D. Perlmutter, editor, Studies in Relational Grammar. Chicago University Press, 1983.

[Borsley, 1987] R. Borsley. Subjects and Complements in HPSG. CSLI, 1987.

[Borsley, 1989] R. Borsley. An HPSG approach to Welsh. Journal of Linguistics, 25:333-354, 1989.

[Gazdar et al., 1985] G. Gazdar, E. Klein, G. Pullum, and I. Sag. Generalized Phrase Structure Grammar. Blackwell, 1985.

[Hinrichs and Nakazawa, 1990] E.

Hinrichs and T. Nakazawa. Subcategorization and VP Structure in German. In Hughes, Shaun, and Salmons, editors, Proceedings of the third Symposium on Germanic Linguistics. Benjamins, 1990.

[Miller and Sag, 1993] P. Miller and I. Sag. French Clitic Movement without Clitics or Movement. Paper presented at the LSA annual meeting and manuscript, 1993.

[Miller, 1992a] P. Miller. Clitics and Constituents in Phrase Structure Grammar. Garland, 1992.

[Miller, 1992b] P. Miller. Postlexical Cliticization vs. Affixation : Coordination Criteria. In C. Canakis, G. Chan, and J. Denton, editors, Proceedings of the 28th Regional Meeting of Chicago Linguistic Society, 1992.

[Monachesi, 1992] P. Monachesi. The Use of Nonlocal Features in the Analysis of Italian Object Clitics and Clitic Climbing. Paper presented at the third CLIN meeting and submitted for the proceedings, 1992

[Monachesi, 1993] P. Monachesi. Restructuring Verbs in Italian HPSG Grammar. In Proceedings of the 29th Regional Meeting of the Chicago Linguistic Society, 1993. To appear.

[Pollard and Sag, 1987] C. Pollard and I. Sag. Information-Based Syntax and Semantics. Fundamentals., volume 1. CSLI, 1987.

[Pollard and Sag, 1993] C. Pollard and I. Sag. HeadDriven Phrase Structure Grammar. CSLI and University of Chicago Press, 1993. To appear.

[Pollard, 1990] C. Pollard. On Head Non-movement. In W. Sijstma and A. van Hork, editors, Proceedings of the Symposium on Discontinuous Constituency, 1990.

[Rizzi, 1982] L. Rizzi. Issues in Italian Syntax. Foris, 1982.

[Sanfilippo, 1990] A. Sanfilippo. Thematic Accessibility in Discontinuous Dependencies. In W. Sijstma and A. van Hork, editors, Proceedings of the Symposium on Discontinuous Constituency, 1990.

[Simpson and Withgott, 1986]

J. Simpson and M. Withgott. Pronominal Clitic
Clusters and Templates. In H. Borer, editor, The Syntax of Pronominal Clitics. Syntax and Semantics., volume 19. Academic Press, 1986.

[Zwicky and Pullum, 1983] A. Zwicky and G. Pullum. Cliticization vs. Inflection: English n't. Language, 59.3:502-513, 1983. 